\title{
Issues in the Diagnosis and Management of Urinary Tract Infections in the Older Adult
}

\author{
Lindsay E. Nicolle
}

Published online: 27 July 2013

(C) Springer Science+Business Media New York 2013

\begin{abstract}
Urinary tract infection is a common problem of elderly populations. Clinical trials addressing urinary infection in the elderly frequently use nonstandardized diagnostic criteria, which compromises the validity of conclusions. Studies of prevention of infection in postmenopausal women in the community consistently report outcomes similar to observations in premenopausal women. Antimicrobial prophylaxis or self-treatment is effective, and cranberry products or probiotics are not beneficial. Critical evaluation of nursing home populations reports no association between nonspecific, nonlocalizing symptoms of any duration and bacteriuria. Optimal antimicrobial use for urinary infection, particularly for residents of long term care facilities, remains challenging. A high proportion of residents in some facilities receive prophylactic therapy to prevent urinary tract infections without evidence to support this practice. Evaluations of multifaceted antimicrobial stewardship programs to improve antimicrobial use in nursing homes report modest benefits.
\end{abstract}

Keywords Urinary infections - Antimicrobial resistance · Bacteriuria - Antimicrobial stewardship · Prophylaxis · Nursing homes $\cdot$ Urethral catheters $\cdot$ Chronic catheter

\section{Introduction}

Urinary tract infection is the most common bacterial infection occurring in older adults. The elderly population is heterogeneous. Considerations with respect to urinary tract infection differ for community and institutionalized populations and,

L. E. Nicolle $(\bowtie)$

Department of Internal Medicine and Medical Microbiology,

Health Sciences Centre, University of Manitoba,

Room GG443 - 820 Sherbrook Street,

Winnipeg, MB R3A 1R9, Canada

e-mail:1nicolle@hsc.mb.ca amongst institutionalized residents, for those with and without chronic indwelling catheters [1, 2]. Asymptomatic bacteriuria is common in all older populations [1-3]. The high prevalence of asymptomatic bacteriuria means a positive urine culture has limited specificity for diagnosis of symptomatic infection, and limitations in clinical ascertainment result in overdiagnosis and overtreatment of symptomatic infection in older adults, particularly residents of long term care facilities [1, 2, 4]. Substantial inappropriate antimicrobial use contributes to emergence of resistant organisms, increased risk of Clostridium difficile colitis, and other adverse antimicrobial effects in these populations.

\section{Community Elderly}

\section{Diagnosis}

Brazilian women over 65 years of age presenting for a first office visit to a general practitioner or nephrologist for any reason were evaluated for urinary infection in a prospective case series at a single university hospital [5]. Potential genitourinary symptoms were systematically recorded and a urine specimen for culture obtained. For the 598 women enrolled (mean age 71.9 years), $14.2 \%$ gave a history of previous urinary tract infection and $21.1 \%$ had significant bacteriuria. Of these, $4.5 \%$ were considered asymptomatic bacteriuria and $16.6 \%$ symptomatic urinary tract infection. In a univariate analysis, women with a "urinary tract infection" were significantly older ( 78 vs. 71.3 years; $p<0.001$ ), had a previous history of urinary infection $(46.5 \%$ vs. $7.8 \% ; p<0.001)$, were diabetic $(23.5 \%$ vs. $18.2 \%$; $p=0.04$ ), and had symptoms of "vaginitis" - not defined $(53.5 \%$ vs. $1.8 \% ; p<0.001)$. As reported in previous studies in postmenopausal women, sexual activity was not a risk factor for bacteriuria or infection. The main urinary "symptom", however, was foul smelling urine, identified in 
$60.6 \%$ of subjects with symptomatic infection, and the sole symptom for $26.3 \%$. This is not a valid symptom for diagnosis of urinary infection in elderly populations, so the clinical criteria used limit the study utility.

Predictive factors for urinary tract infection were identified in a population based prospective study of very elderly Dutch subjects initially enrolled at 86 years of age in Leiden and followed prospectively [6•]. Two-thirds of 479 subjects were female and $22 \%$ were resident in long-term care facilities. The definition for urinary tract infection was "when treating physicians diagnosed urinary infection based on signs and symptoms and urinalysis". This definition is nonspecific, so the diagnostic ascertainment is problematic. The incidence of urinary infection reported was $11.2 / 100$ person years for all subjects (12.8/100 women; 7.8/100 men). A multivariate analysis of subjects who lived independently identified predictive factors for urinary infection of: history of urinary infection between the age of 85 and 86 years (hazard ratio 3.3; $95 \%$ CI 2.1, 5.3), impaired cognitive function (MMSE < 19) (HR 1.9; 1.1, 3.4), disability in daily living (HR $1.9 ; 1.3,2.9$ ), self-reported urine incontinence (HR 1.7; 1.2, 2.6), and unintentional loss of feces (HR 2.7; 1.6, 4.8). The risk factors identified in this very old community population are similar to those reported for urinary infection or asymptomatic bacteriuria in residents of long-term care facilities.

The clinical presentation of urinary infection in older compared with younger Israeli women was described in a prospective cohort study [7]. Patients enrolled had presented to a community clinic and were diagnosed with a urinary infection based on one or more symptoms of frequency, urgency, hematuria, painful voiding and urination, new onset incontinence, or pain in the lower abdomen together with a urine culture which grew $\geq 10^{3} \mathrm{cfu} / \mathrm{ml}$ of a uropathogen. Thus, all study subjects had localizing genitourinary complaints at enrollment. Women were excluded if symptoms had been present for more than 5 days, with pyelonephritis, with complicating genitourinary factors, or with antibiotics given within the past month. There were 94 postmenopausal women (mean age 69.2 years) and 102 premenopausal (48.1 years). "Generalized unspecific" symptoms, classified as cold, chills, constipation, diarrhea and "storage symptoms" were more common in postmenopausal than premenopausal women. However, as all women had localizing genitourinary symptoms, the relevance of these nonlocalizing symptoms for urinary infection in the absence of an uninfected age matched control group, is not clear.

These reports highlight the uncertainties of the clinical and microbiological diagnosis of urinary tract infection in elderly women. Two of the three studies used nonspecific, unvalidated definitions for clinical symptoms which would promote overdiagnosis of symptomatic infection in individuals with positive urine cultures. The third study concluded that post-menopausal women with localizing genitourinary symptoms were more likely to have "nonspecific symptoms" but the specificity of these symptoms for urinary infection was not explored. An additional concern is the variability in quantitative criteria for a positive urine specimen. Marques [5] required $\geq 10^{5} \mathrm{cfu} / \mathrm{ml}$, Caljouw [6•] did not confirm infection with culture, and Arinzon [7] accepted quantitative counts $\geq 10^{3} \mathrm{cfu} / \mathrm{ml}$. A quantitative count of $<10^{5} \mathrm{cfu} / \mathrm{ml}$ for the diagnosis of symptomatic infection in a voided specimen is validated only for cystitis in premenopausal women. Lower quantitative counts likely represent contamination for most elderly women, particularly those who are functionally impaired and where a high prevalence of asymptomatic bacteriuria is anticipated. Advancing the knowledge of urinary infection in elderly populations requires valid, standardized, clinical and microbiologic diagnostic criteria. Clinical trials should use restrictive criteria with a high probability of identifying true urinary infection (i.e., localizing genitourinary symptoms and $\geq 10^{5} \mathrm{cfu} / \mathrm{ml}$ of a uropathogen).

\section{Prevention}

Several strategies for prevention of urinary infection in postmenopausal women have been explored. Zhong [8] randomized 68 postmenopausal Chinese women, in a nonblinded study, to receive low dose antibiotic nightly or "single dose antibiotic each time they experienced conditions predisposing to UTI". These conditions included working or walking for greater than 2 hours, travelling, "emiction holdback", sexual intercourse, diarrhea, and constipation. The risk of urinary tract infection associated with any of these activities has not been quantified, with the exception of sexual intercourse which has consistently been reported not to be a risk factor for infection for postmenopausal women. It is unclear whether the subjects randomized to intermittent therapy were self-treating for perceived early symptoms of urinary tract infection or, in fact, using antimicrobial prophylaxis following risk activities. After 12 months, the rates of urinary infection were similar in the two groups -1.4 and 1.9 per patient, and significantly less than the 4.7 and 5.1 per person-year, respectively, reported prior to enrollment. Antibiotic use in the intermittent group was only $9.5 \%$ that of the continuous prophylaxis group. Thus, less frequent prophylaxis or self-treatment may be effective for postmenopausal women, but the triggers for self-medication need to be more precisely characterized.

A randomized, placebo controlled, double blind study enrolled female Japanese outpatients aged 20-79 years (median 57 years) and compared the efficacy of one bottle of cranberry juice daily versus placebo for prevention of urinary infection [9]. After 24 weeks there was no difference in 
a per-protocol analysis for the overall "cumulative nonrelapse rates" between the two groups ( $\log$ rank test $p=0.4209$ ). However, in a non-prespecified subgroup analysis, women over 50 years who received cranberry juice had significantly fewer episodes of acute uncomplicated cystitis $(p=0.0425)$. The diagnosis of symptomatic urinary infection was, however, not rigorous, as it was based on patient interview at clinic attendance every 4 weeks without microbiologic confirmation. Thus, this study is not definitive in addressing the efficacy of cranberry juice for prevention of infection in older women.

Beerepoot et al. [10••] reported a randomized, doubleblind noninferiority trial of trimethoprim/sulfamethoxazole (TMP/SMX) prophylaxis once daily or oral capsules of Lactobacillus rhamnosus GR-1 and $L$. reuteri $\mathrm{RC}-14$ twice daily to prevent recurrent urinary infection in 252 postmenopausal women in the Netherlands. The study subjects had a very high frequency of infection prior to enrollment - an average of 6.9 episodes per year. In addition, $43 \%$ of study subjects had complicated infection, a population for whom antimicrobial prophylaxis has never previously been shown to be effective. Criteria for the diagnosis of symptomatic urinary infection was subject report of symptoms. Microbiologically confirmed symptomatic infection was a secondary outcome. During 12 study months, at least one symptomatic urinary infection occurred in $69.3 \%$ of the TMP/SMX recipients and $79.1 \%$ of the Lactobacillus spp. participants $(p=0.02)$, with a median time to first infection of 6 and 3 months $(p=0.02)$, respectively. Thus, the probiotic arm did not meet noninferiority criteria. TMP/SMX appeared to be more effective than Lactobacilli spp. in preventing uncomplicated infection [mean clinical recurrences $1.9(1.4-2.6)$ vs. $3.2(2.5-4.2)]$, but not for complicated infections $(4.4 ; 3.4-5.7$ vs. $3.4 ; 2.6-4.3)$. There was an increased frequency of isolation of TMP/SMX resistant $E$. coli strains from the fecal flora of women randomized to the antibiotic arm.

These studies of interventions to prevent urinary infection in postmenopausal women report outcomes consistent with those previously reported in studies enrolling premenopausal women. Continuous or intermittent antimicrobial prophylaxis or self-treatment are effective, and prophylactic antimicrobial strategies should likely be tailored to the specific patient. Cranberry products or probiotics were not beneficial for preventing urinary infection in postmenopausal women.

\section{Long Term Care Facilities}

\section{Clinical Diagnosis}

Administrative data from the ED component of the 20012008 United States national hospital ambulatory medical care survey were analyzed to describe symptoms of patients diagnosed with urinary tract infection [11]. There were records for 5 million older adults, including 2.2 million over 85 years of age. Localizing genitourinary symptoms were recorded for $32 \%$ of all adults diagnosed with urinary infection, $24 \%$ of adults $65-84$ years, and $17 \% \geq 85$ years. The diagnosis of urinary infection in nursing home residents correlated with increased fever (odds ratio $1.63 ; 1.18,2.25$ ) and altered mental status (OR 4.79; 0.99, 3.82) at presentation, but significantly fewer lower urinary symptoms were recorded (OR $0.35 ; 0.22,0.55)$. The authors concluded that fever and urinary tract symptoms are not recorded for most adults over 65 years of age diagnosed with urinary infection, and that diagnostic criteria for urinary infection specific for use for older adults in the acute care setting need to be developed and validated.

A cross sectional study of 651 elderly men and women resident in 32 nursing homes in Sweden correlated the presence and duration of nonspecific symptoms with bacteriuria [12••]. Symptoms evaluated included restlessness, fatigue, confusion, aggressiveness or "not being herself/himself", as well as potential localizing urinary symptoms of dysuria, urgency and fever, and were stratified by present for less than one week, more than one week but less than one month, and more than one month but less than 3 months. The attending nurse at the home identified and recorded symptoms and obtained a urine specimen for culture. Bacteriuria was present in $32 \%$ of subjects; $22 \%$ had E. coli isolated, $3.8 \%$ Klebsiella pneumoniae, and $2.6 \%$ Enterococcus faecalis. The only clinical variable that correlated with $E$. coli bacteriuria was "not being herself/himself within 3 months" and, for any bacteriuria, confusion, fatigue and not being herself/himself that occurred or changed within 3 months. However, none of these symptoms had clinical utility to identify bacteriuria in a positive/negative etiological predictive value analysis. The author's conclusion that urine cultures provide little or no useful information for evaluating nonspecific, non localizing symptoms among elderly residents of nursing homes is consistent with previous studies which have systematically addressed this question.

Das et al. [13••] stratified subjects enrolled into a previously reported study of symptoms associated with bacteriuria and pyuria in institutionalized elderly women by the number of episodes identified for a given subject during the 12 study months. In a multivariate analysis, the number of episodes correlated with antibiotic use (OR 30.57; 15.98-58.49) and isolation of multiply drug resistant gram negative rods from urine specimens (OR 1.91; 1.14-3.18), but not with change in mental status or hospitalization for urinary tract infection. Thus, the adverse outcomes observed were those attributable to antimicrobial use rather than urinary infection itself.

A nonrandomized pilot study of 29 male and 45 female incontinent residents in six Taiwan nursing homes evaluated whether increased fluid intake had an impact on bacteriuria 
[14]. Subjects were managed with either maintenance of their current fluid regimen (30 subjects) or increased fluid intake (44 subjects), as recommended by the nursing administrator. The prevalence of bacteriuria at enrollment was $29.7 \%$ for the study cohort and this decreased to $17.6 \%$ at 6 weeks. A higher proportion of bacteriuric subjects with increased fluid intake cleared bacteriuria $-15.9 \%$ vs. $6.7 \%$ $(p=0.052)$. The authors concluded that further evaluation of the impact of increased fluid intake on bacteriuria in incontinent residents was warranted.

Eighty American female nursing home residents with a history of urinary infection were enrolled into a pilot study of the effect of daily cranberry capsules to decrease the prevalence of bacteriuria with pyuria [15•]. There were four study arms - one, two, or three cranberry capsules or placebo capsules daily. The primary outcome was bacteriuria with pyuria at days 7, 14, 21 and 28 days, with the outcome reported as a compilation of urine cultures from all followup assessments. The prevalence of $E$. coli bacteriuria plus pyuria for one, two or three cranberry capsules was $40.3 \%$, $29.9 \%$ and $34.3 \%$, respectively. For bacteriuria with any organism, the proportions were $45.8 \%, 42.9 \%$, and $50.7 \%$. Placebo recipients had a prevalence of $E$. coli bacteriuria of $43.4 \%$ and for any bacteriuria of $50 \%$, so there was no suggestion of an effect of cranberry capsules on bacteriuria.

\section{Residents with Chronic Catheters}

All residents with chronic indwelling urinary catheters in 78 nursing homes of one Swedish county were surveyed with urine cultures in March 2010 to identify bacterial flora and the antimicrobial susceptibility of the strains isolated [16]. Susceptibility of these isolates was compared with that of bacteria isolated from urine specimens submitted from hospitals and primary health care in the same geographic area during the first 6 months of 2010. Significant bacteriuria was defined as $\geq 10^{4} \mathrm{cfu} / \mathrm{ml}$ for pure culture or $\geq 10^{5} \mathrm{cfu} / \mathrm{ml}$ for mixed culture. There were 126 men (mean age 85 years) and 37 women (mean age 88 years) surveyed, and $7 \%$ were receiving antimicrobials when the urine specimen was obtained. Nine $(5.5 \%)$ patients had had a chronic catheter for less than 3 months, 37 (22.7\%) for 3-12 months, 44 (27\%) for 13-24 months, and 73 (44.8\%) for over 24 months. Bacteriuria was present in 159 of 163 urine samples $(98 \%$ ); three of the four samples without bacteriuria were obtained from patients receiving antimicrobials. The most common bacterial species isolated were Enterococcus spp. (46\%), E. coli (54\%), Pseudomonas aeruginosa (8\%), and Proteus mirabilis (12\%). E. coli were isolated more frequently from women, and P. mirabilis from subjects with more prolonged catheterization. The $E$. coli strains isolated were more resistant to ciprofloxacin than the comparator strains (16.9\% versus $7.9 \%)$.
A controlled trial in a single Taiwanese long term care facility randomized 60 men and women with chronic indwelling catheters to receive either $100 \mathrm{ml}$ diluted rice vinegar or $100 \mathrm{ml}$ of water by nasogastric tube each day for 4 weeks [17]. The hypothesis was that the $3-5 \%$ acetic acid in the vinegar would decrease urinary $\mathrm{pH}$ and this would inhibit bacteriuria. A new catheter was inserted when the study was initiated, urinalysis was obtained weekly, and urine cultures collected initially and on day 28 . There was a significant decline in urine $\mathrm{pH}$ and turbidity observed in the vinegar treated subjects, but no significant difference in bacteriuria between groups. Three patients in the control group and none in the experimental group had symptomatic urinary tract infection, but the study was not blinded and the definition of symptomatic urinary tract infection was "physician diagnosed", so of uncertain precision.

\section{Antimicrobial Use for Urinary Tract Infection}

\section{Antimicrobial Use}

Administrative data were analyzed to compare the quality and pattern of use of antibiotics given for urinary infection between institutionalized and community dwelling elderly persons $(\geq$ 65 years) in Sweden [18]. In a one-day point prevalence survey, $1.6 \%$ of institutionalized elderly subjects and $0.9 \%$ of those in the community were receiving antibiotics for urinary infection. Of these, $15 \%$ of institutional and $19 \%$ of community subjects were receiving fluoroquinolones. The proportions of women treated with pivmecillinam, nitrofurantoin, or trimethoprim, the antimicrobials recommended in the Swedish guidelines, were $29 \%, 27 \%$ and $45 \%$, respectively, amongst the institutionalized and $40 \%, 28 \%$ and $34 \%$ in the community elderly. The authors concluded that current recommendations for therapy were not being followed in elderly populations in the community or long-term care facilities, and further critical evaluation of antimicrobial treatments was necessary.

Antibiotic prescribing in nursing homes in Norway [19], a country with a low prevalence of antimicrobial resistance, was described in a retrospective record review of residents of all nursing homes in one county between March 2007 and February 2008. There were 714 antimicrobial courses prescribed to 327 residents, for a period prevalence of $6.6 \%$. Of these, $378(53 \%)$ were for urinary tract infection. Trimethoprim and pivmecillinam accounted for $68 \%$ of antimicrobial prescriptions given for a diagnosis of urinary infection. Compliance with national guidelines was $77 \%$ for this indication compared with $79 \%$ for respiratory infections and $76 \%$ for skin and soft tissue infections. They concluded that active implementation of guidelines for antibiotic use would be necessary to improve antimicrobial prescribing. 
Another study from Norway reports a one day point prevalence study in 44 nursing homes undertaken in April-May 2006 to describe characteristics of prophylactic treatments given for urinary infection [20•]. Of the 1,473 residents, $18 \%$ were receiving one or more agents for prophylaxis of urinary infection, but the proportion varied from $0-50 \%$ among different nursing homes. Methenamine (48\% of prescriptions), vitamin C (32\%), and cranberry products $(10 \%)$ were the most common. Trimethoprim and nitrofurantoin were given for prophylaxis to $5 \%$ and $4 \%$ of the residents receiving any prophylactic therapy. The authors concluded that therapy to prevent urinary infection in Norwegian nursing homes is frequently prescribed despite there being no evidence to support this practice.

The presence of an indwelling urinary catheter was the strongest predictor of antibiotic treatment of asymptomatic bacteriuria in a cross-sectional study of residents in four nursing homes in central Texas [21]. There were 23 prescriptions given for urinary infection to residents with an indwelling catheter and 19 (82.6\%) of these episodes were not accompanied by acute or localized symptoms. For residents without a catheter, almost $50 \%$ of prescriptions for suspected urinary infection were given without documented localizing symptoms. About three-quarters of the antibiotic courses were ordered after laboratory results became available to clinicians, suggesting asymptomatic bacteriuria was being treated. The only statistically significant variable which correlated with an antimicrobial prescription for asymptomatic bacteriuria was the specific nursing home of residence. The authors concluded that improving antimicrobial use in nursing homes requires addressing the common practice of clinical decisions being made solely on the basis of diagnostic testing without supporting clinical evidence.

There is increasing attention to antimicrobial use in longterm care residents given the high levels of antimicrobial resistance observed in many of these facilities. These recent studies confirm the previously recognized problems of inappropriate antimicrobial treatment for asymptomatic bacteriuria, high levels of use of prophylaxis for urinary infection, and antimicrobial use inconsistent with guidelines. The reports also highlight a wide variability in inappropriate use among different institutions $(20,21)$, an observation which suggests that institution-specific review and interventions are necessary to address inappropriate antimicrobial use.

\section{Antimicrobial Stewardship in LTCF}

Rummukainen [22••] described a multifaceted intervention in long term care facilities in Finland developed specifically to address inappropriate antimicrobial prophylaxis for urinary infection. The intervention included a team of infectious diseases consultant, infection control nurse, and geriatrician who visited all long term care facilities in central Finland during
2004-2005. The site visits incorporated a structured interview discussing specific patients, identification of ongoing systemic antimicrobial use, and discussion of appropriate diagnostic practices for urinary infection. Regional guidelines for prudent use of antimicrobials in long term care facilities were developed. The proportions of residents receiving any antimicrobial prophylaxis was $19.9 \%, 16.9 \%, 16.2 \%$ and $15.4 \%$ in 2005 , 2006, 2007 and 2008, respectively; and $66.6 \%-81.1 \%$ of this use was for urinary infection. Following the intervention, the proportion of residents receiving antimicrobial prophylaxis for urinary infection decreased from $13 \%$ to $6 \%$, a statistically significant decline.

A cluster randomized controlled trial in Swedish nursing homes evaluated the effectiveness of a multifaceted educational intervention targeting nurses and physicians to improve antimicrobial prescribing for residents [23•]. The intervention included small educational group sessions with nurses and physicians, feedback on antimicrobial prescribing, and provision of guidelines and written materials. The primary outcome was the proportion of women prescribed a fluoroquinolone for lower urinary tract infection. Secondary outcomes were antibiotics prescribed for all infections, number of urinary infections per resident, proportion of all infections treated with an antibiotic, proportion of infections handled by physicians as "wait and see" and, for lower urinary infection in women, the proportion treated with nitrofurantoin. Forty-six homes completed the study. After 2 years there was no difference between the intervention and control homes, although the use of fluoroquinolones for urinary infection declined significantly for both. There were, however, significant differences which favored the intervention homes in total antibiotics given for all infections and number of episodes managed by "wait and see". The authors concluded that the intervention had a modest effect and, while the primary outcome of fluoroquinolone use for urinary infection decreased significantly, this decrease could not be attributed to the intervention. A country-wide antimicrobial use program had been initiated in Sweden while the study was ongoing and may have promoted changes in fluoroquinolone use in all study homes.

These studies report some success in improving antimicrobial use with the implementation of strategies targeting specific prescribing practices relevant to urinary infection at the institutional level. However, optimal approaches to improve antimicrobial use for urinary infection or other indications in long term care facilities are not yet well defined. Further evaluation of single and multifaceted interventions to identify those which are most beneficial and cost effective is necessary.

\section{Antimicrobial Resistance}

A retrospective and prospective cross-sectional study identified male patients over 18 years with community acquired 
E. coli febrile urinary tract infection who attended an emergency department at a Spanish hospital between January 2008 and January 2011 [24]. The 153 men had a mean age of 60.7 years; 6 were nursing home residents. Independent associations with isolation of fluoroquinolone resistant $E$. coli in a multivariate analysis were health care acquired urinary tract infection (OR 3.82; 1.3-11.24), antibiotic treatment within the previous 30 days (OR $5.85 ; 2.3-14.88$ ), and prior fluoroquinolone treatment $(13.97 ; 2.73-71.53)$.

Tinnelli et al. [25] described characteristics of Italian long term care facility residents with extended-spectrum betalactamase (ESBL) producing organisms isolated from urine culture. A case-control study compared 99 residents with ESBL positive gram negative rod urinary infections, 99 residents with gram negative bacteriuria without ESBL strains, and 99 residents without gram negative organisms isolated. Patients with ESBL producing organisms more frequently had a chronic urinary catheter (OR 15; 6.9$30.5)$ and had received antimicrobial therapy within the prior 30 days (OR 4; 2.2-10.9). After adjusting for type, dose and duration of antibiotic exposure, 7 or more days of exposure to a fluoroquinolone or third generation cephalosporin predicted isolation of ESBL producing organisms.

The optimal treatment of some highly resistant gram negative organisms is not yet well defined. Neuner et al. [26] described a case series of 41 hospitalized patients with urinary tract infection with multiply resistant organisms treated with fosfomycin. The mean age was $62 \pm 13$ years and $45 \%$ were male. The 44 organisms included 13 carbapenemase-resistant K. pneumoniae (KPC), 8 P. aeruginosa, 7 VRE, 7 ESBL producers, and 9 others. In vitro susceptibility to fosfomycin was $86 \%$. The average number of fosfomycin doses given was $3.3 \pm 1.2$, and $27 \%$ of the patients received combination therapy with other antimicrobials. Microbiologic cure was $59 \%$, with $24 \%$ relapses and $17 \%$ reinfections. Cure rates by pathogen were KPC $46 \%$, P. aeruginosa $38 \%$, VRE $71 \%$, ESBL $57 \%$ and others $100 \%$. Resistance to fosfomycin developed following therapy in the bacterial isolates of 3 patients with kidney transplants and ureteral stents. Alexander [27] reported a retrospective cohort study of treatment and clinical outcomes of urinary infection caused by KPC producing Enterobacteriaceae in 21 patients with a mean age of 62.4 years; $71 \%$ were female and $15 \mathrm{had}$ an indwelling urinary catheter. Antimicrobials with in vitro susceptibility were given to 14 of the 21 patients. Successful outcomes were ultimately achieved in 16 (76\%) patients. All 7 patients who received aminoglycoside therapy were cured. Other antimicrobials given included tigecycline, minocycline and fosfomycin. Tasbakan [28•] treated with nitrofurantoin 45 females and 30 males in Turkey with ESBL producing nitrofurantoin-sensitive $E$. coli isolated from lower urinary tract infection. The mean age was $54 \pm 17$ years, and all but 14 subjects had complicated infection. At $7-9$ days, clinical success was $69 \%$ and microbiologic success $68 \%$. At $28-$
31 days post therapy, $6.5 \%$ had reinfection and $3.2 \%$ relapse. These case series which include a high proportion of older individuals provide preliminary observations addressing the potential efficacy of antimicrobial treatments for highly resistant uropathogens.

\section{Conclusions}

Clinical trials of urinary infection in elderly populations are often compromised by failure to use standard and validated definitions for diagnosis of symptomatic urinary tract infection. For nursing home residents, nonlocalizing signs or symptoms do not correlate with bacteriuria. These observations are consistent with previous reports and support current recommendations for initiation of antimicrobial treatment for urinary infection in residents without indwelling catheters only when acute, localizing, genitourinary findings are present. Strategies for prevention of urinary infection in younger women are also valid for postmenopausal women resident in the community. Excess and inappropriate antimicrobial use for urinary tract infection continues to be described in long term care facilities, and use of prophylaxis for urinary infection is a particular concern. Implementation of multifaceted antimicrobial stewardship programs may improve inappropriate antimicrobial use in some long term care facilities. However, diagnosis, antimicrobial resistance, and antimicrobial use for urinary infection in long term care facilities remains problematic.

\section{Compliance with Ethics Guidelines}

Conflict of Interest Lindsay E. Nicolle has received compensation from Johnson \& Johnson, Cerexa, and LEO for serving as a consultant, and she received payment for preparation of a manuscript from Johnson \& Johnson.

Human and Animal Rights and Informed Consent This article does not contain any studies with human or animal subjects performed by any of the authors.

\section{References}

Papers of particular interest, published recently, have been highlighted as:

- Of importance

-. Of major importance

1. Nicolle LE. Urinary tract infections in the elderly. Clin Geriat Med. 2009;25:423-36.

2. Beveridge LA, Davey PG, Phillips G, McMurdo MET. Optimal management of urinary tract infections in older people. Clin Interv Aging. 2011;6:173-80. 
3. Ariathianto Y. Asymptomatic bacteriuria. Prevalence in the elderly population. Aust Fam Physician. 2011;40:805-9.

4. Buhr GT, Genao L, White HK. Urinary tract infections in longterm care residents. Clin Geriatr Med. 2011;27:229-39.

5. Marques LPJ, Flores JT, Barros Jr O, Rodrigues GB, Mourao C, Moreira RMP. Epidemiological and clinical aspects of urinary tract infection in community-dwelling elderly women. Braz J Infect Dis. 2012;16:436-41.

6. Caljouw MAA, den Elzen WPJ, Cools HJM, Gussekloo J. Predictive factors of urinary tract infections among the oldest in the general population. A population-based prospective follow-up study. BMC Med. 2011;9:57. This prospective population based study describes the frequency of urinary infection in a cohort of subjects over 85 years of age. Independent predictors of urinary infection in community subjects were functional impairment and prior urinary infection, similar to observations in long term care facility residents.

7. Arinzon Z, Shabat S, Peisakh A, Berner Y. Clinical presentation of urinary tract infection (UTI) differs with aging in women. Arch Gerontol Geriatr. 2012;55:145-7.

8. Zhong YH, Fang Y, Zhou JZ, et al. Effectiveness and safety of patient-initiated single-dose versus continuous low-dose antibiotic prophylaxis for recurrent urinary tract infections in postmenopausal women: a randomized controlled study. J Int Med Res. 2011;39:2335-43.

9. Takahashi S, Hamasuna R, Yasuda M, et al. A randomized clinical trial to evaluate the preventive effect of cranberry juice (UR65) for patients with recurrent urinary tract infection. J Infect Chemother 2012; Sep 8. (Epub ahead of print).

10. $\bullet$ Beerepoot MAJ, ter Riet G, Nys S, Stobberingh E, Geerlings SE. Lactobacilli vs antibiotics to prevent urinary tract infections. Arch Intern Med. 2012;172:704-12. For post-menopausal women with frequent recurrent urinary tract infection, trimethoprim/sulfamethoxazole prophylaxis was superior to oral probiotic capsules for prevention. This benefit was restricted to women with uncomplicated urinary tract infection.

11. Caterino JM, Ting SA, Sisbarro SG, et al. Age, nursing home residence, and presentation of urinary tract infection in U.S. emergency departments, 2001-2008. Acad Emerg Med. 2012;19:117380 .

12. •• Sundvall P-D, Ulleryd P, Gunnarsson RK. Urine culture doubtful in determining etiology of diffuse symptoms among elderly individuals: a cross-sectional study of 32 nursing homes. BMC Fam Pract. 2011;12:36. This study examined the correlation of bacteriuria with a wide range of nonspecific symptoms of varying duration among nursing home residents. No symptoms had prognostic value for identifying bacteriuria. It was concluded that obtaining urine cultures in these individuals was unhelpful for clinical management.

13. •- Das R, Towle V, Van Ness PH, Juthani-Mehta M. Adverse outcomes in nursing home residents with increased episodes of observed bacteriuria. Infect Cont Hosp Epidemiol. 2011;32:84-6. An increasing number of episodes of bacteriuria with pyuria identified in female residents was associated with increased antimicrobial use and isolation of multiply drug resistant gram negative rods, but not with changes in mental status or hospitalization for urinary infection. The adverse events were those of inappropriate antimicrobial use rather than attributable to bacteriuria.

14. Lin S-Y. A pilot study - fluid intake and bacteriuria in nursing home residents in southern Taiwan. Nursing Res. 2013;62:66-72.

15. - 15. Bianco L, Perrelli E, Towle V, et al. Pilot randomized controlled dosing study of cranberry capsules for reduction of bacteriuria plus pyuria in female nursing home residents. Jour Amer Ger Soc 60:1180-1181. This pilot study reported no decrease in bacteriuria in residents given any of three doses of cranberry capsules compared with placebo.
16. Jonsson K, Claesson BEB, Hedelin H. Urine cultures from indwelling bladder catheters in nursing home patients: a point prevalence study in a Swedish county. Scand J Urol Nephrol. 2011;45:265-9.

17. Chung Y-C, Chen H-H, Yeh M-L. Vinegar for decreasing catheterassociated bacteriuria in long-term catheterized patients: a randomized controlled trial. Biol Res Nurs. 2012;14:294-301.

18. Haasum Y, Fastbom J, Johnell K. Different patterns in use of antibiotics for lower urinary tract infection in institutionalized and home-dwelling elderly: a register-based study. Eur J Clin Pharmacol. 2013;69:665-71.

19. Fagan M, Maehlen M, Lindbaek M, Berild D. Antibiotic prescribing in nursing homes in an area with low prevalence of antibiotic resistance: Compliance with national guidelines. Scand J Prim Health Care. 2012;30:10-5.

20. • Bergman J, Schjott J, Blix HS. Prevention of urinary tract infections in nursing homes: lack of evidence-based prescription? BMC Geriatr. 2011;11:69. A point prevalence survey in Norwegian nursing homes reported that $18 \%$ of residents were receiving prophylaxis for urinary infection, despite there being no evidence to support prophylactic use in this setting.

21. Phillips CD, Adepoju O, Stone N, et al. Asymptomatic bacteriuria, antibiotic use, and suspected urinary tract infections in four nursing homes. BMC Geriatr. 2012;12:73.

22. • Rummukainen J-L, Jakobsson A, Matsinen M, et al. Reduction in inappropriate prevention of urinary tract infections in long-term care facilities. Am J Infect Control. 2012;40:711-4. The authors developed and tested a multifaceted intervention to decrease the use of prophylactic antimicrobials for urinary infection in Finnish long term care facilities. There was a significant decrease in the proportion of residents receiving antimicrobial prophylaxis for urinary infection, which was attributed to the intervention program.

23. - Pettersson E, Vernby A, Holstad S, Lundborg CS. Can a multifaceted educational intervention targeting both nurses and physicians change the prescribing of antibiotics to nursing home residents? A cluster randomized controlled trial. J Antimicrob Chemother. 2011;66:2659-66. The primary outcome of fluoroquinolone use for urinary infection decreased for both intervention and control homes, but there was no difference post-intervention between the two. Overall antimicrobial use and a "wait and see" approach for infection management were significantly improved in intervention homes.

24. Smithson A, Chico C, Ramos J, et al. Prevalence and risk factors for quinolone resistance among Escherichia coli strains isolated from males with community febrile urinary tract infection. Eur J Clin Microbiol Infect Dis. 2012;31:423-30.

25. Tinelli M, Cataldo MA, Mantengoli E, et al. Epidemiology and genetic characteristics of extended-spectrum $\beta$-lactamaseproducing Gram-negative bacteria causing urinary tract infections in long-term care facilities. J Antimicrob Chemother. 2012:67:2982-7.

26. Neuner EA, Sekeres J, Hall GS, van Duin D. Experience with fosfomycin for treatment of urinary tract infections due to multidrug-resistant organisms. Antimicrob Agents Chemother. 2012;56:5744-8

27. Alexander BT, Marschall J, Tibbetts RJ, et al. Treatment and clinical outcomes of urinary tract infections caused by KPCproducing Enterobacteriaceae in a retrospective cohort. Clin Ther. 2012;34:1314-23.

28. • Tasbakan MI, Pullukcu H, Sipahi OR, Yamazhan T, Ulusoy S. Nitrofurantoin in the treatment of extended-spectrum $\beta$-lactamase producing Escherichia coli-related lower urinary tract infection. Int J Antimicrob Agents. 2012;40:554-6. In patients with ESBL producing organisms sensitive to nitrofurantoin isolated from urinary culture, $70 \%$ were cured with nitrofurantoin therapy. Most of these patients had complicated lower urinary tract infection. 